\title{
Averaging for High Fidelity Modeling-Toward Large Eddy Simulations in Multi-Passage Multi-Row Configurations
}

A major challenge in high-fidelity turbomachinery flow computations is the need for high resolution in a very large domain of multiple blade-passages and multiple blade rows. Scale-resolving turbulent solutions are prohibitively costly. The question is, what will it take to get high-fidelity solutions if we are only after time-mean flows for aerothermal performance?

A novel two-scale approach is adopted to address the issue by coupling between a local fine-mesh domain in a single-passage and a global coarse-mesh multi-passage domain. This is achieved by harnessing the extra product terms generated when averaging a nonlinear process in time, as well as in space. As such a space-time averaging is purposely applied in either a direct mode or an inverse one in the two domains respectively. The source terms in a compact form (one scalar for one equation) are conveniently obtained to enable the interaction between the single-passage fine-mesh and the multi-passage coarse-mesh solutions. The converged solution for this two-scale coupled system should meet two seemingly conflicting requirements: an otherwise poorly conditioned local fine-mesh domain is now subject to a right environment/flow conditions, and an otherwise poorly resolved global coarse-mesh domain is now effectively subject to high resolution. In this paper, the concepts, the formulations of the framework methodology, and the implementation methods will be described. The validity and feasibility of the approach for efficient scale-resolving high-fidelity turbomachinery flow simulations will be illustrated by several computational examples of practical interest. [DOI: 10.1115/1.4049616]

Keywords: computational fluid dynamics (CFD), turbomachinery blading design

\section{Introduction}

Computational fluid dynamics (CFD) has become indispensable in modern turbomachinery design, research, and development. Advanced CFD methods have been continuously pursued in terms of higher resolution numerical methods, higher fidelity flow modeling, and more consistent boundary condition treatment. The rapid increase of computing power has enabled a step-change in CFD modeling fidelities to be adopted for more realistic configurations in aviation and power generation industries. It is noted however that the methods and models developed, tested, and applied for external flows (e.g., those for aircraft wings) tend to be directly applied to turbomachinery configurations with a certain "time lag," chiefly because of the extra computational resources (compared to the external flow counterparts) required for larger computational domains consisting of multiple blade passages and multiple blade rows. As such, turbomachinery flows are commonly regarded merely as some more expensive, though straightforward, applications for higher fidelity CFD simulations. A question of interest is, can we also harness the fundamental characteristics of turbomachinery flows to facilitate high-fidelity modeling development and applications? Before attempting to address this, we shall first consider past and current turbomachinery CFD developments, as overviewed in the following. The objectives, specific context, and scope of the present work will then be introduced.

Contributed by the International Gas Turbine Institute (IGTI) of ASME for publication in the Journal of Turbomachinery. Manuscript received September 3, 2020; final manuscript received October 26, 2020; published online February 1, 2021. Assoc. Editor: Dr. David G. Bogard.
1.1 Overview of Turbomachinery Computational Fluid Dynamics Development

1.1.1 Steady Reynolds-Averaged Navier-Stokes Model. Although typical turbomachinery flow is inherently unsteady due to the relative motion between rotating and stationary blade rows, a single blade passage can be taken as a base unit domain, where a steady flow in the relative frame of reference with a blade-to-blade periodicity can be assumed. From the viewpoint of moving from a complete circumferentially averaged through-flow analysis to a $3 \mathrm{D}$ passage-resolving CFD solution in the 1960s-70s, this basic steady blade-to-blade passage model should be, as it had been, more than adequately acceptable. The flow modeling fidelities have advanced rapidly and successfully from an inviscid Euler flow model [1] to various forms of Reynolds-averaged Navier-Stokes (RANS) viscous flow models (e.g., Refs. [2-4]). Some of the most fundamental understanding of the 3D flow physics at a blade-passage level has been gained by the use of the single-passage steady RANS methods, aided with experimental validations. Over years, turbulence closure models have also been adopted as they were advanced (e.g., the one-equation model [5] and the two-equation model [6]), so have various RANS-based transition models.

In the context of making use of the distinctive features of turbomachinery flow to advance turbomachinery CFD, two impactful pieces of previous efforts are particularly worth noting, which are related to the key theme of the present work, "Averaging."

(i) The mixing-plane method for multiple blade rows instigated by Denton $[7,8]$ : The mixing-plane treatment has made a profound impact on the design (as well as on research) of all types of working turbomachines. The success of the mixingplane method underscores the importance and power of simple overall conservation as applied to an averaged flow, 
even with detailed unsteady disturbances being missed completely.

(ii) The average-passage approach instigated by Adamczyk $[9,10]$ : Though still based on a steady flow framework, the approach is developed to capture the influence of periodically passing non-uniform flows of blade passages in an adjacent relatively moving blade row. In essence, the framework deals with the nonlinear interaction between the mean-flow and the non-uniform (steady/unsteady) disturbances, thus is a manifestation of the general Reynolds-averaging (for turbulence as we know it) in a turbomachinery context. The formulations of Adamczyk are effectively a modeling effort of closing the extra nonlinear product terms due to the averaging, as for turbulence.

It is quite remarkable that although turbomachinery is inherently unsteady, it is these steady RANS methods in general and the mixing-plane model in particular which have been so far the primary workhorse in turbomachinery blade aerothermal designs and analyses.

1.1.2 Unsteady Reynolds-Averaged Navier-Stokes Model. With the rate of increase in computing power in the past 30 years, the unsteady Reynolds-Averaged Navier-Stokes (URANS) model should be a natural candidate to "succeed" the RANS given the inherent unsteady nature of turbomachinery flows. The situation has however turned out somewhat differently. It is recognized that URANS has been widely used in blade aeromechanics/ aeroelasticity applications, e.g., Refs. [11-13]. This wide utilization may in no small part be attributed to the need to match the flow conditions of these aeroelastic analyses to their design base flows, which are typically obtained by steady RANS. It is also noted that URANS has been widely adopted in the research of unsteady flow phenomena of relatively long temporal and spatial length scales such as inflow distortions, e.g., Refs. [14-16] and rotating stall/rotating instabilities, e.g., Refs. [17,18].

On the other hand, URANS methods seem to have made far less impact on basic aerodynamic and aerothermal design applications, than originally expected. Recognizing the extra computing resource required, we may also note a relevant issue related to the modeling consistency of URANS. Making RANS time-accurately unsteady does lead to a seemingly higher modeling fidelity per se, but at the same time raises the potential modeling inconsistency in terms of the resolved unsteadiness versus the modeled unsteadiness. Consider for instance the self-excited vortex shedding, as for practical high-pressure turbine and compressor blades with blunt trailing edges. The onset and strength of vortex shedding is strongly influenced by the upstream turbulent boundary layer. For a RANS model, the local vortex shedding around a blade trailing edge can also be strongly dependent on the way the upstream turbulence is modeled, e.g., Ref. [19]. The URANS model should only be regarded as consistent when there is a clear frequency spectral gap between the modeled and resolved unsteadiness. For a trailing-edge vortex shedding, the scales of the shed vortices may well be comparable to those of large-scale turbulence fluctuations. In this case, when a RANS solver becomes time-resolved, the selfexcited unsteadiness (vortex shedding and/or turbulence) can become both modeled and resolved, thus being "double counted." It may be argued that the risk of "double-counting" unsteadiness presents an extra challenge to URANS for aerodynamic and aerothermal applications, regardless of computational costs.

1.1.3 High-Fidelity Scale-Resolving Turbulence Simulations. It is widely accepted that the single most important factor in the accuracy and uncertainty of current CFD applications is turbulence modeling. Thus, moving from entirely modeled treatments (RANS) to scale-resolving solutions, e.g., large-eddy simulations (LES) for turbulence is naturally expected as computing power increases. The overall trend is evident from the number of LES oriented ASME/ IGTI publications in the last 10 years, e.g., for compressors and turbines [20-22]. There are also some efforts with direct numerical simulations (DNS), e.g., Refs. [23,24]. The interest in advancing scale-resolving models seems to be overwhelming, so much so that a large number of the recent developments in turbomachinery CFD have progressed from the RANS to the LES/DNS directly by skipping the URANS phase altogether. This model fidelity "jump" might be to some extent attributed to the potential modeling inconsistency of URANS, as discussed earlier.

The huge rise in the computational cost associated also clearly presents a challenge to the LES for high Reynolds number conditions of practical interest. Various negotiations and compromises between modeling fidelity and cost have been undertaken for practical applications. A notable example is the detached eddy simulations (DES) after Spalart [25] and similar hybrid treatments between a scale-resolving outer flow and a RANS modeled near-wall region, where the mesh count/resolution requirement scales strongly with the Reynolds number. It is recognized that hybrid RANS-LES/ DES type methods have attracted a lot of attention and seem to be quite successful in making LES possible for practical configurations. It should be observed with caution that the hybrid switch of the modeling fidelity from the resolved to the modeled one is inherently empirical and, in particular, tends to take place in a region with high turbulence intensity and high gradient. Thus, the near-wall flow parameters of interest (e.g., wall surface friction, heat transfer) may be subject to considerable empiricism. An additional complication is that the RANS models have been developed for steady flows but the near-wall region in a hybrid treatment will inevitably be subject to the unsteadiness of the scale-resolving outer flow region, thus leading to extra uncertainty in the near-wall region due to "double-counting" of turbulence unsteadiness.

In the context of the present interest, upgrading the modeling fidelity for turbomachinery flow will have to face the challenge due to the inherent unsteady and interactive multi-passage multirow environment. Consider one single blade-passage domain. A typical mesh count for a RANS is around $1 \times 10^{6}$ whilst its counterpart for an LES will be $50-100 \times 10^{6}$. This rise in computing cost per passage is already very large. A further increase by $1-2$ orders of magnitude due to multi-blade passages (30-70 per row) would make it prohibitively expensive.

Given the significant cost implication of eddy-resolving turbulence solutions, considerations have also been given on how to improve the flow modeling fidelity based on a RANS/URANS framework. It is noted that there have been active recent research activities in the Machine-Learning improved RANS (ML-RANS) models (e.g., Refs. [26,27]). Given the data-driven nature of the ML-based models, a good ML-RANS will need a large amount of high-fidelity results/data. This again points to the need for faster and more affordable high-fidelity solutions. And in the context of turbomachinery, these high-fidelity solutions will need to include multi-passage multi-row interactions.

1.2 Aims and Scope of Present Work. Given the state of art and the corresponding issues of interest as outlined in the overview earlier, the present work is aimed at developing an efficient multipassage model for scale-resolving turbulent flow simulations and doing so at a cost comparable to that of the same modeling fidelity for a single-passage domain. The primary intent is to achieve this through a local-averaging-based approach. The main considerations of the work are specifically influenced by three previous efforts:

(i) Adamczyk's average-passage model $[9,10]$ underlines how extra stress terms can be made use of to account for the nonlinear interaction between a mean-flow and non-uniform disturbances. This effectively is a "turbomachinery manifestation" of the Reynolds averaging.

(ii) The work by Ning and He [19] shows that a steady-like solution for time-averaged flow with self-excited (nonexternally driven) unsteadiness may be obtained by making use of the corresponding unsteady stress terms. 
(iii) Denton's integral body force form [2] summing up all viscous stress terms. The treatment underscores the fundamental manner in which the viscous effects are at play in balancing each flow equation for conservation, regardless of whatever flow modeling fidelity is adopted.

The present work is built on a recent effort by He and Yi [28], where a new two-scale framework is adopted in conjunction with an ensemble-averaging procedure to split deterministic and nondeterministic unsteady disturbances, demonstrated largely for 2D cases. The present work extends the $\mathrm{He}$ and $\mathrm{Yi}$ framework without the distinction between non-deterministic and deterministic unsteadiness. Consequently, it will now be possible to have a finemesh solution only in one single blade passage. Also, the present work deals with two blade rows, rather than a single row as in the previous work.

\section{Framework Concepts and Definitions}

We start by considering two contrasting scenarios of CFD solutions in general:

(a) On the one hand, we can easily solve a truncated small domain with a fine mesh and high-fidelity model, but it would be of no use under uncertain environment/boundary conditions.

(b) On the other hand, we can easily have well-defined environment/boundary conditions for a large global domain, but the solution would also be of no use if poorly resolved with a too coarse mesh and/or inadequately low fidelity flow modeling.

If we are after a solution of high fidelity as well as high resolution for a large global domain, a direct approach covering all scales will be costly. To deal with the general issue, here we adopt a two-scale approach. The basic idea is to have a high-fidelity well-resolved local region in a single-passage domain and to "propagate" the effect of the local high resolution to the otherwise poorly resolved global multi-passage domain. As such, the global domain will be subject to an equivalent high resolution. To this end, two main aspects need to be considered.

First, to facilitate the propagation of the local fine-mesh resolution effect from a single-passage to a global multi-passage domain, we intend to make use of the similarity of the passage-passage flow patterns. Given a seemingly random instantaneous unsteady turbulent flow in a rotor-stator stage with unequal rotor-stator blade count, e.g., Fig. 1(a), there does not appear to be any directly definable passage-passage similarity. However, looking at its time-averaged counterpart, we will get a very different picture. In fact, for a rotor-stator stage, there is a simple direct passage-passage periodicity (Fig. 1(b)). Also, note that the time-averaged flow is spatially much smoother. Thus, even when we deal with the unsteady flow with deterministic and non-deterministic disturbances, we shall seek to work through the averaged flow.

Second, we are reminded that a local fine-mesh solution of a truncated domain on its own would be wrong as it is subject to a wrong environment, no matter how high the resolution is. The global coarse-mesh domain solution on its own would also be wrong, given its poor resolution. To reach a correct overall solution, the method will have to include the interaction between the local and global domains so that the final converged solution will meet the two requirements at the same time: the local fine-mesh domain is now subject to the right environment, and the global coarse-mesh domain is now equivalently subject to a high resolution.

The following sections will describe the building blocks for a proper averaging and propagation of the local high-resolution effect to a global domain, in order to include the interactions between the two domains and between the two scales.

2.1 Governing Equations With Lumped Viscous Terms. The three-dimensional compressible unsteady Navier-Stokes
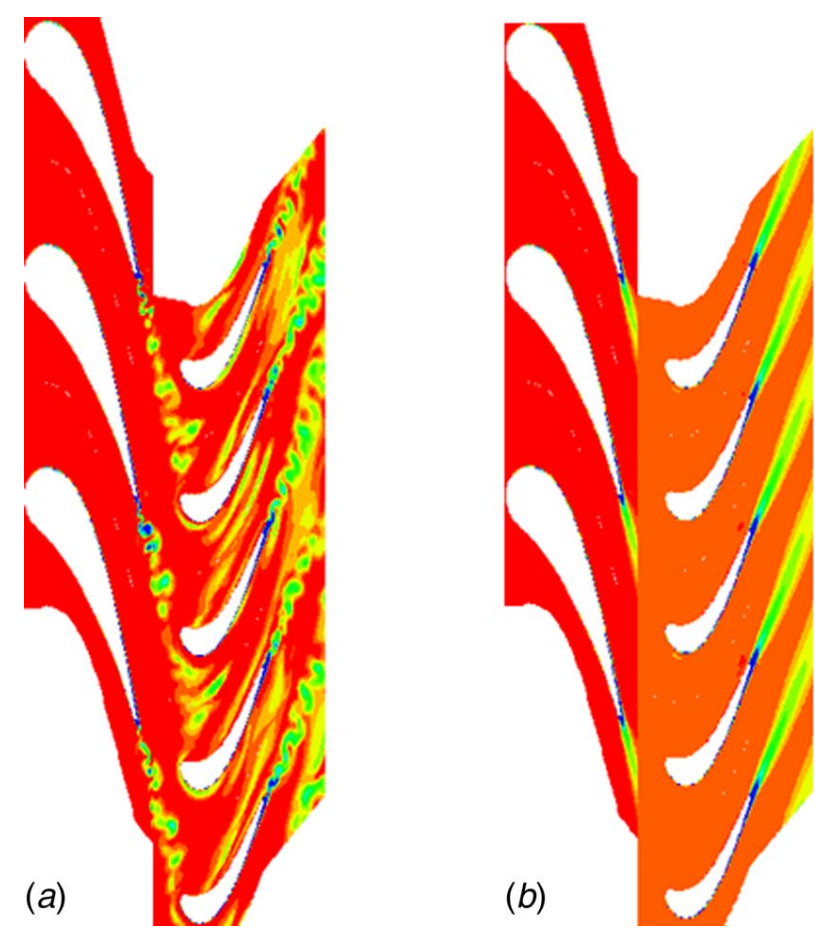

Fig. 1 Entropy contours (unsteady versus time-averaged): (a) instantaneous and (b) time-averaged

equations are adopted in an absolute frame of reference in a cylindrical coordinate system for turbomachinery flows

$$
\begin{gathered}
\frac{\partial \rho}{\partial t}+\frac{\partial \rho u}{\partial x}+\frac{\partial \rho\left(v-v_{g}\right)}{r \partial \theta}+\frac{\partial \rho w r}{r \partial r}=0 \\
\frac{\partial \rho u}{\partial t}+\frac{\partial\left(\rho u^{2}+p\right)}{\partial x}+\frac{\partial \rho u\left(v-v_{g}\right)}{r \partial \theta}+\frac{\partial \rho u w r}{r \partial r}=\frac{\partial \tau_{x x}}{\partial x}+\frac{\partial \tau_{x \theta}}{r \partial \theta}+\frac{\partial r \tau_{x r}}{r \partial r} \\
\frac{\partial \rho v r}{\partial t}+\frac{\partial \rho v u r}{\partial x}+\frac{\partial \rho v r\left(v-v_{g}\right)+p r}{r \partial \theta}+\frac{\partial \rho v w r}{r \partial r}=\frac{\partial r \tau_{x \theta}}{\partial x}+\frac{\partial r \tau_{\theta \theta}}{r \partial \theta}+\frac{\partial r \tau_{\theta r}}{r \partial r} \\
\frac{\partial \rho w}{\partial t}+\frac{\partial \rho w u}{\partial x}+\frac{\partial \rho w\left(v-v_{g}\right)}{r \partial \theta}+\frac{\partial\left(\rho w^{2}+p\right) r}{r \partial r}=\frac{\partial \tau_{r x}}{\partial x}+\frac{\partial \tau_{r \theta}}{r \partial \theta}+\frac{\partial r \tau_{r r}}{r \partial r} \\
\frac{\partial \rho e}{\partial t}+\frac{\partial \rho H u}{\partial x}+\frac{\partial \rho H\left(v-v_{g}\right)}{r \partial \theta}+\frac{\partial \rho H w r}{r \partial r}=\frac{\partial\left(u \tau_{x x}+v \tau_{x \theta}+w \tau_{x r}+q_{x}\right)}{\partial x} \\
+\frac{\partial\left(u \tau_{x \theta}+v \tau_{\theta \theta}+w \tau_{r \theta}+q_{\theta}\right)}{r \partial \theta}+\frac{\partial r\left(u \tau_{x r}+v \tau_{\theta r}+w \tau_{r r}+q_{r}\right)}{r \partial r}
\end{gathered}
$$

where $v_{g}$ is the local grid velocity for a mesh domain fixed with a rotor row. After all viscous stress and heat flux terms are expressed in terms of the corresponding velocity and temperature gradients, we intentionally lump all right-hand side (RHS) together to a single scalar for each of the momentum and energy equations

$$
\begin{gathered}
\frac{\partial \rho u}{\partial t}+\frac{\partial\left(\rho u^{2}+p\right)}{\partial x}+\frac{\partial \rho u\left(v-v_{g}\right)}{r \partial \theta}+\frac{\partial \rho u w r}{r \partial r}=s_{x} \\
\frac{\partial \rho v r}{\partial t}+\frac{\partial \rho v u r}{\partial x}+\frac{\partial \rho v r\left(v-v_{g}\right)+p r}{r \partial \theta}+\frac{\partial \rho v w r}{r \partial r}=s_{\theta} \\
\frac{\partial \rho w}{\partial t}+\frac{\partial \rho w u}{\partial x}+\frac{\partial \rho w\left(v-v_{g}\right)}{r \partial \theta}+\frac{\partial\left(\rho w^{2}+p\right) r}{r \partial r}=s_{r}
\end{gathered}
$$




$$
\frac{\partial \rho e}{\partial t}+\frac{\partial \rho H u}{\partial x}+\frac{\partial \rho H\left(v-v_{g}\right)}{r \partial \theta}+\frac{\partial \rho H w r}{r \partial r}=s_{e}
$$

The lumped form of viscous terms underlines a simple but very useful point: regardless of the modeling fidelity for viscous terms (from a completely empirical correlation to a full differential form of viscous stresses), what matters for each flow equation is the scalar sum of all viscous stress terms. It holds true regardless of the detailed distributions and breakdowns of all these viscous stresses, heat, and work fluxes. This fundamental feature will be further utilized in the present framework.

2.2 Averaging for a Modeling Purpose. "Averaging," a seemingly simple term, seems to be associated with many original ideas and considerations with far-reaching impact for modeling (e.g., Refs. [7-10]), as well as for meaningfully consistent data processing [29].

We first take a slightly different look at the conventional timeaveraging in the context of Reynolds-averaging for turbulent flow before extending the formulations to a spatial and temporal averaging.

2.2.1 Time Averaging. Write the full flow equation system (Eq. (1)) in a simple form denoting $U$ as the vector of conservative flow variables

$$
\frac{\partial U}{\partial t}+R(U)=0
$$

A standard temporal decomposition is given by

$$
U(x, t)=\bar{U}(x)+U^{\prime}(x, t)
$$

Time-averaging the flow equations, we must then have

$$
\overline{R(U)}=0
$$

Due to the nonlinearity of the advective fluxes, $\overline{R(U)} \neq R(\bar{U})$, a well-known consequence of the Reynolds averaging. We now purposely express the time-averaged flow equations in two modes. The first one is the common form used for turbulence closure, notionally labeled here as a "direct mode"

Reynolds Averaging ("Direct Mode"):

$$
\frac{\partial U}{\partial t}+R(U)=S_{t}
$$

$S_{t}$ is the vector of five scalar elements representing the lumped Reynolds stresses and energy fluxes (in the same form as Eq. (2), with the first element being zero for the mass continuity). When the solution to Eq.( 6) converges to a steady-state, it should produce the time-averaged flow field as intended, if correct $S_{t}$ can be provided. The direct mode of the Reynolds-averaging underlines its basic objective that the averaged flow field can now be solved with a much coarser resolution. But in doing so, the otherwise closed flow equation system becomes open. Thus, a correct averaged flow solution with the coarser resolution can only be obtained if the extra nonlinear product terms can be found to balance the equations. The latter has been the motivation of all turbulence modeling developments with a varying extent of empirical ingredients. It is worth emphasizing that Eq. (6) underlines the general fundamental working for any turbulence models. A turbulence model, regardless of its exact form (e.g., a simple algebraic mixing-length or an extensive differential form of the Reynolds stress transport), will ultimately affect the flow solution by balancing the flow equations with one scalar for each equation. Basically, the "direct mode" treats the nonlinear product source terms as input to solving the time-averaged flow.

On the other hand, we write the time-averaged equation the other way around. We then have the second mode (which has so far been rarely considered), labeled as an "inverse mode"
Reynolds Averaging ("Inverse Mode"):

$$
S_{t}=R(\bar{U})
$$

Equation (7) means that if we happen to have a given target timeaveraged solution $(\bar{U})$ of high fidelity and high resolution (regardless of the origin), we can get those lumped scalars (one for each equation) for $S_{t}$, simply by computing the corresponding flux residuals explicitly and noniteratively using the flow variables of the target solution. As such, these scalars can be used as the source term to drive a new solution to reproduce the target. This property in itself does not seem to be of any practical use, but it will feature very differently when the inverse mode is used as part of an interactive procedure to be introduced later.

2.2.2 Space-Time Averaging. Reynolds-averaging shows how a meaningfully averaged solution of high resolution in time can be obtained through a method with low resolution (or no resolution at all) in time. We now extend this useful temporal treatment to space in seeking to establish a spatially as well as temporally averaged flow solution through a method with low resolution in both space and time.

Consider a two-scale mesh system consisting of

(a) Coarse (RANS) mesh: this is for getting an adequately accurate solution which can be obtained if the scalars for the lumped turbulence stress/flux terms are provided.

(b) Fine (LES) mesh: we build a fine LES mesh by subdividing a coarse "RANS" mesh. A "RANS" coarse-mesh cell then consists of a locally embedded LES mesh block.

For a spatial and temporal decomposition, the time-averaging is carried out as usual over the entire time period of the solution. The spatial averaging is carried out locally for each coarse-mesh cell. Given that the fine LES mesh cells are created by simply subdividing a coarse-mesh cell, the local spatial average is defined as a volumeweighted average of flow variables for each coarse-mesh cell. In a discrete form, a flow variable is identified by its corresponding finemesh cell index $m$. The instantaneous spatial average for coarsemesh cell $M$ of total volume $V_{M}$ at time-step $n$ becomes

$$
\tilde{U}(M, n)=\frac{1}{V_{M}} \sum_{m=1}^{N_{m}} U(m, n) v_{m}
$$

$N_{m}$ is the number of fine-mesh cells with volume of $v_{m}(m=1, \ldots$, $N_{m}$ ) contained in coarse-mesh cell $\mathrm{M}$. The corresponding space-time average for coarse-mesh cell $M$ is then

$$
\overline{\tilde{U}}(M)=\frac{1}{N_{t}} \sum_{n=1}^{N_{t}} \tilde{U}(M, n)
$$

$N_{t}$ is the total number of time-steps with a constant physical timestep size being assumed. Note that the space-time averaging is a simple sum of corresponding instantaneous variables in time and in space weighted by the constant time-step and constant mesh volumes. Thus $\bar{U}$ is effectively a space-averaging of corresponding time-averaged fine-mesh variables. It is usefully reminded that a time-averaged flow is much smoother than its instantaneous counterpart (Fig. 1). Therefore, no extra filtering is considered necessary for spatial averaging.

Space-time averaging the unsteady flow equations (Eq. (3)), we have $\overline{R(U)}=0$. But again, due to the nonlinearity of advective fluxes, we also have $R(\overline{\tilde{U}}) \neq 0$. Therefore, extra terms will be needed to balance the flow equations with the space-time averaged flow variable $\overline{\tilde{U}}$, similarly to the Reynolds-averaged flow equations (Eq. (6)).

Now, denote the coarse-mesh domain with subscript " $C$ " and the fine-mesh domain with subscript " $f$." We can then see how a direct mode and an inverse mode of the space-time averaged equations can be illustratively made use of in the two domains accordingly. 
Space-time averaging ("Direct Mode"): The unsteady flow equations applied to the global coarse-mesh domain can be expressed as

$$
\frac{\partial U_{C}}{\partial t}+R_{C}\left(U_{c}\right)=S_{s t}
$$

Equation (10) means that a coarse-mesh domain solution can be driven toward a target by the space-time source term $\left(S_{s t}\right)$ generated elsewhere (in the present work, it will be generated from the local fine-mesh solution as introduced in the following). Similar to the Reynolds-averaging, when Eq. (10) converges to a steady-state, the coarse-mesh solution should produce a meaningful space-time averaged flow field, as long as proper source terms $S_{s t}$ can be provided.

\section{Space-time averaging ("Inverse Mode”):}

$$
S_{s t}=R_{C}\left(\overline{\tilde{U}}_{f}\right)
$$

Equation (11) will be used to generate the source terms from a target space-time averaged fine-mesh solution $\overline{\tilde{U}}_{f}$. The source terms will then be used to drive the global coarse-mesh domain to a solution equivalently with a high resolution of the local fine-mesh domain.

The combined applications of the "direct mode" and the "inverse mode" of the space-time averaging (Eqs. (10) and (11)) in the two domains respectively underscore the inter-dependence between the two domains as the key ingredient of the approach:

(a) If the source terms in Eq. (10) (one scalar for each equation in each mesh cell) can be provided for a global coarse-mesh domain, a converged coarse-mesh solution should then correspond to that as if the fine meshes were applied for the whole global domain.

(b) If a global coarse-mesh is enabled to provide a right base flow environment, any truncated local fine-mesh domains within the global domain would then be able to produce a meaningful local solution of high resolution under right boundary conditions.

Note again that neither of the two parts on its own would be correct. Therefore, the global coarse-mesh domain and the local fine-mesh region will have to be coupled. The space-time average source term $\left(S_{s t}\right)$ should act as the linkage in the coupling between the coarse and fine-mesh domains.

Before ending this section on the framework concepts, some further comments should be made about the local space-averaging as defined in Eq. (8). A question we should face is, what does this space-averaging actually mean or imply? It is helpful to draw a parallel between the Reynolds-averaging (time-averaging) and the present local spatial averaging. It may be easy to see that a timeaveraged state is a notional representation of a series of timedependent states, so a time-averaged state is by itself not directly a physical state. Similarly, we should stress that a spatially averaged state is not a physically mixed-out state either. A mix-out state has to go through a physical mixing process (thus with some associated entropy generation) whilst the averaged state as a notional representation should not have any mixing loss. In a similar vein, when we seek a solution to a locally averaged flow field, its meaningfulness should be viewed similarly to that of a Reynolds-averaged flow state.

\section{Method for Implementation}

3.1 Unsteady Navier-Stokes Solver. The baseline flow solution method adopted is an in-house multi-block code which the author has developed and updated over many years [30-32]. Flow is governed by the three-dimensional unsteady compressible Navier-Stokes equations. The computational domain is discretized with a multi-block structured mesh. The equations are spatially discretized using a cell-center-based finite volume scheme. The spatial discretization for the advective fluxes is based on the pressure-convective flux split upwinding discretization, advection upstream splitting method (AUSM), Liou [33]. The baseline implementation of the AUSM is second-order accurate in space for a uniformly regular mesh, following van Leer's MUSCL formulation. A relatively simple extension to a third-order spatial accuracy has been implemented, following Cada and Torrilhon [34], through a reformulation of the nonlinear limiter. The full viscous dissipation terms are discretized using the second-order central difference as applied in a finite volume scheme.

The inlet/exit far-field boundary conditions can be set by the compatibility conditions: the specified inlet stagnation pressure and temperature, inflow angle, and exit static pressure. Alternatively, the local characteristics-based non-reflective boundary conditions can be used. On a solid surface, a nonslip wall condition is used for a viscous wall or a symmetry condition for an inviscid boundary. A rotor-stator interface is subject to the direct slidingplane treatment.

The discretized equations are explicitly integrated in time by a four-stage Runge-Kutta scheme. The physical temporal derivatives are discretized by a second-order backward difference in the form of the dual-time-stepping after Jameson [35]. The Runge-Kutta scheme is used to march in the pseudo-time to converge the solution at each physical time-step. The pseudo time marching (subiterations) can be accelerated by the local time-stepping and multigrid. Options for accelerating unsteady solutions also include a pointwise implicit updating.

The baseline solver can be run in a RANS/URANS mode with two options for turbulence closure: the one-equation SpalartAllmarus model [5] and the $k-\omega$ SST two-equation model of Menter [6]. For large-eddy simulations, the Smagorinsky model is implemented for closing the sub-grid scales. However, in the present calculations, the explicit sub-grid model is not applied in order to avoid its interference with the prediction of laminarseparation-related transition. As such, the present scale-resolving turbulence calculations can be regarded as an implicit LES, where the sub-grid scales are dampened by inherent numerical dissipations of the AUSM discretization.

3.2 Dual Meshes With Local Embedded Fine Mesh. Start with a base coarse-mesh for a global multi-passage domain (Fig. 2(a)). It is intended that the base mesh, though generally coarse, should be sufficiently fine so that transport of large-scale disturbances, and interactions among blade passages and blade rows will be captured. For practical interest, we can take the global base mesh domain with its mesh density and resolution comparable to those of typical RANS. It is in this context that a base
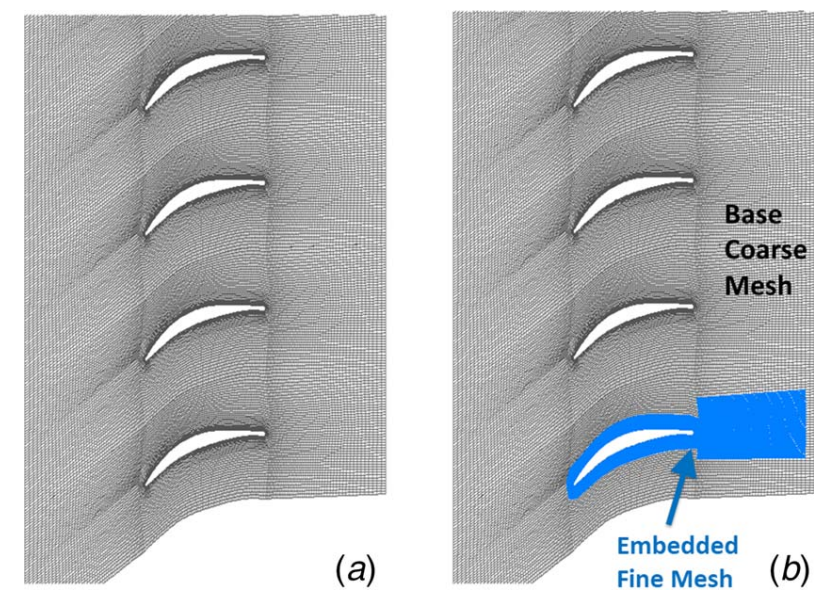

Fig. 2 Dual meshes with embedded fine-mesh block: (a) base coarse-mesh and $(b)$ with embedded fine-mesh 
mesh may be labeled as a "RANS" mesh, though a "STANS" mesh should more appropriately reflect the space-time averaged nature of the target base-mesh solution.

The fine-mesh domain is generated by embedding locally a finemesh block in the base coarse-mesh. This equivalently generates the fine-mesh cells by subdividing a coarse-mesh cell. Given that the main interest is to apply a high-fidelity scale-resolving model in the turbulence dominated regions, the first candidate of the local embedded fine-mesh for LES is the near-wall region for a wallresolved LES. In this case, only one blade passage in a multipassage domain needs to be embedded with a fine mesh (e.g., around the bottom blade in Fig. 2(b)).

There are several advantages of using the locally embedded dual meshing. Firstly, a local refinement for anticipated or inspected high gradient regions can be quite easily carried out. It also enables convenient enforcement of flux conservation at coarse-fine domain interfaces and boundaries. The one-to-one fine-coarse mesh correspondence leads to easy implementation of the space-time averaging, and a straightforward residual evaluation to generate the source terms from the local fine-mesh blocks, to be passed on to the global coarse-mesh region.

The price of the dual meshing appears to be the overhead in using both the coarse mesh and the fine mesh in the same place for those embedded regions. Take a typical RANS mesh as the coarse mesh and a typical LES mesh for the fine mesh, for example. Knowing that an LES mesh count per blade passage would typically be about two orders of magnitude higher than that of a RANS, we will then find the overhead cost of the doubly meshed setup (e.g., with a $3 \mathrm{D}$ coarse-mesh cell embedded by $5 \times 5 \times 5$ fine-mesh cells) to be easily justifiable.

\subsection{Two Domain Solutions and Scale Interaction}

3.3.1 Flow Solution of Embedded Fine-Mesh Domain. For the local embedded domain, the fine-mesh solution is essentially a direct time-accurate unsteady Navier-Stokes solution. The main extra consideration and treatment needed are to do with the interface between the fine-mesh domain and the adjacent coarse-mesh one. Given that the fine-mesh blocks are designed to be embedded in the regions with high flow gradients, the interfaces between the fine- and coarse-mesh domains should thus be located in regions with low flow gradients.

Figure 3 gives a local view of mesh cells around an interface, showing the hanging mesh nodes and cells of the fine-mesh and their adjacent coarse-mesh cells. For the present cell-center-based finite volume discretization, the flow variables at the dummy mesh cell-center points outside the fine-mesh domain $\left(U_{i n t}\right)_{f}$ are interpolated by the flow variables $\left(U_{\text {int }}\right)_{C}$ at the centers of adjacent coarse-mesh cells

$$
\left(U_{\text {int }}\right)_{f}=f_{i}\left[\left(U_{\text {int }}\right)_{C}\right]
$$

where $f_{i}[$ ] denotes a local linear interpolator. The flux conservation for the interface boundary is enforced by overwriting the

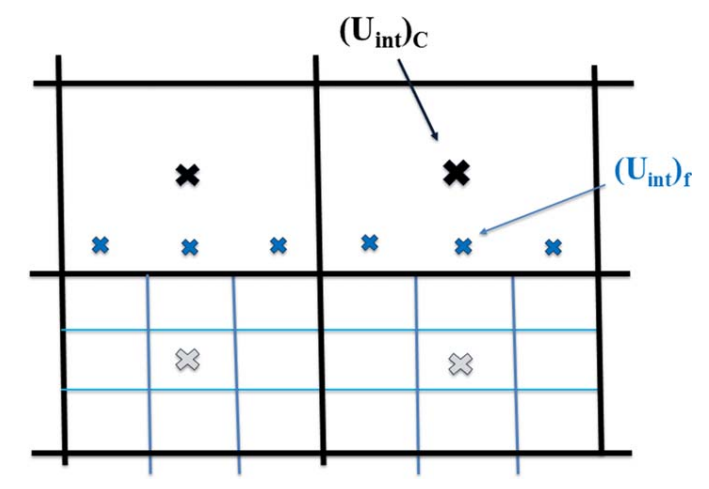

Fig. 3 Mesh cells adjacent to domain Interface coarse-mesh boundary face fluxes completely by the sum of the fluxes of the corresponding fine-mesh boundary faces.

The large spatial length scale disparity also provides an opportunity for a time-marching with extra multi-stepping at a small timestep to enhance temporal resolution for the fine-mesh blocks locally. When time marching in a large time-step for the coarse-mesh region, we can take a number of fractional steps to time-march in the fine-mesh blocks. The boundary dummy points of the fine-mesh can then be interpolated from the corresponding coarse-mesh boundary points and between two large time instants.

For a stator-rotor interface, the embedded fine-mesh also provides a useful means of generating fine-mesh resolved turbulence fluctuating inflow disturbances for a downstream blade row. Inflow unsteady flow disturbances at the downstream side of an interface for those coarse-mesh passages can be constructed by an instantaneous circumferential Fourier spectrum [36] from the single-passage fine-mesh solution. The low harmonics (deterministic disturbances) are taken from the local coarse-mesh spatial Fourier spectrum. The high harmonics are taken from the embedded fine-mesh passage with the corresponding phase-shift to align with the local wakes of the coarse-mesh passages. Thus, all passages of the downstream blade row are subject to instantaneous inflow wake fluctuating disturbances effectively of the fine-mesh resolution.

3.3.2 Source Term Generation From Fine-Mesh Domain. The function of the source terms is to drive the global non-embedded coarse-mesh domain to a solution equivalent to having a local embedded fine-mesh. To this end, the target solution of the coarsemesh domain is the fine-mesh solution when temporally and spatially averaged on the corresponding coarse-mesh cells. In addition, as discussed earlier around Fig. 1, we should only propagate timeaveraged parameters from passage to passage. Hence, we generate source terms from the space-time averaged flow solution in the local fine-mesh domain.

First, we need to perform the averaging during the time-marching solution. This is carried out in a moving-average (e.g., Refs. [31,32]). At time-step $N$, the corresponding space-time averaged for a coarse-mesh cell with local fine-mesh cells embedded is

$$
\overline{\tilde{U}}_{f}^{N}=\frac{N-1}{N} \overline{\tilde{U}}_{f}^{N-1}+\frac{1}{N} \tilde{U}_{f}^{N}
$$

This is a very straightforward updating, and we only need to store the space-time averaged in the coarse-mesh cells for one time-step. The influence of initial solution transients can also be reduced by taking a different starting-point counting time-steps, to speed up reaching the final time-averaged state. Having updated the equivalent coarse-mesh target solution $\left(\overline{\tilde{U}}_{f}\right)$, we then simply use the inverse mode (Eq. (11)) of the space-time averaging to noniteratively obtain the source term $S_{s t}$.

3.3.3 Coarse-Mesh Solution With Source Term. The function of the coarse-mesh domain solution is to drive its flow field to a target from the corresponding space-time averaged fine-mesh solution $\left(\overline{\tilde{U}}_{f}\right)$. The source terms generated from Eq. (11) will be sufficient only if the coarse-mesh domain converges to a steady solution. For general unsteady flows resolved in the coarse-mesh domain, we will also need to include extra source terms due to timeaveraging the coarse-mesh unsteady solution [32]. The resultant coarse-mesh equation becomes

$$
\frac{\partial U_{C}}{\partial t}+R_{C}\left(U_{C}\right)=S_{s t}+\left(S_{t}\right)_{C}
$$

The extra time-averaging related source term $\left(S_{t}\right)_{C}$ for the coarsemesh domain is given by

$$
\left(S_{t}\right)_{C}=\overline{R_{C}\left(U_{C}\right)}-R_{C}\left(\bar{U}_{C}\right)
$$

The first term on RHS of Eq. (15) is calculated by time-averaging the net fluxes of the coarse-mesh cell, and the second term by the net 
fluxes calculated using the time-averaged coarse-mesh flow variables. To reduce a close local correlation between the local timeaveraged and instantaneous solutions, the extra time-averaging source terms in Eq. (15) are evaluated at the corresponding coarsemesh cells of adjacent blade passages and updated with an underrelaxation.

Assuming the existence of a unique time-averaged state, we should have $\overline{\overline{R_{C}\left(U_{C}\right)}}=\overline{R_{C}\left(U_{C}\right)}$. Time-averaging the unsteady flow equation for a coarse-mesh cell (Eq. (14)), we shall then have the resultant equation

$$
R_{C}\left(\overline{\tilde{U}}_{f}\right)-R_{C}\left(\bar{U}_{c}\right)=0
$$

Thus, the time-averaged coarse-mesh solution $\left(\bar{U}_{c}\right)$ should approach the time-space averaged fine-mesh solution $\left(\overline{\tilde{U}}_{f}\right)$, as intended.

Figure 4 shows the solution flowchart, indicating the main parts of the coupling between the two domains reflecting the corresponding two-scale interaction.

\section{Case Examples for Illustration}

The following numerical test cases are examined to assess the baseline flow solver for LES solutions and the validity of the present approach.

4.1 Large-Eddy Simulations for Compressor Cascade With Separation. The first test case is selected to assess the baseline method capability in performing large-eddy simulations. A lowspeed compressor cascade is selected here. The blade geometry is of a controlled diffusion (CD) blading developed and initially tested by Sanger and Shreeve [37]. The blade profile was later used in an oscillating cascade experiment by Yang and He [38] at a Reynolds number of $2.5 \times 10^{5}$ (based on inlet flow velocity and blade chord length). Under this flow condition, the experimental data indicate a laminar separation around mid-chord on the suction surface followed by a transition to turbulence, and the separated flow becomes reattached. The separation bubble is clearly identifiable in steady and unsteady pressure measurements [38]. The blade geometry also features a relatively blunt trailing-edge; thus, the blade profile loss is also subject to the interaction between the suction surface laminar-separation-related transitional flow and the trailing-edge vortex shedding. In the experiment, the inlet duct flow is subject to a rapid cross-sectional variation with a contraction ratio of 7.5:1 [38]. Thus, the inlet free-stream turbulence is deemed to be negligibly small.

For the present density-based time-marching flow solver, the computations are performed at a higher flow velocity than the experimental condition, still being kept low enough to be within the incompressible flow regime, whilst the experimental Reynolds number is matched.

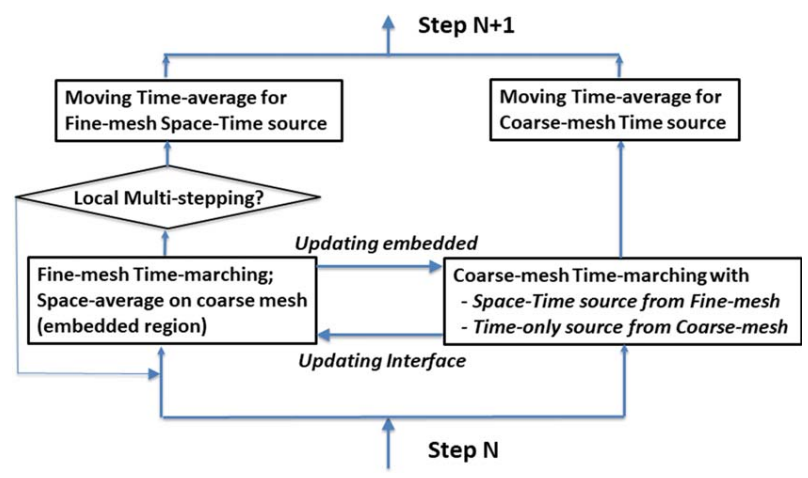

Fig. 4 Flowchart for two-scale coupled solution during each time-step
For a scale-resolving solution, LES computations need to be carried out in a full 3D mode. A single-passage computational domain mesh for a 10\% span sector is shown in Fig. 5. A direct periodicity is applied at the domain boundaries both in the blade pitchwise and in the spanwise directions. The numerical sensitivity is examined for two mesh densities ("Mesh 1" and "Mesh 2") and two time-step sizes ( $1 \mu \mathrm{s}$ and $0.5 \mu \mathrm{s})$, as summarized in Table 1 .

The two meshes examined should correspond to a range of relatively lower mesh count $\left(30-50 \times 10^{6}\right.$ mesh cells/blade passage) for LES. The dual-time-stepping is carried out with 20-30 subiterations per time-step. A passage through-flow time takes about 3000 steps when a physical time-step of $1 \mu$ s is taken.

The scale-resolving capability is consistently indicated by the iso-surface plots of the Q-criterion (Fig. 6). A mid-chord separation on the suction surface is shown at around $40 \%$ chord with a subsequent transition to turbulent flow. This agrees with the experiment (based on the surface pressures to identify the separation bubble). Also interestingly, there appears to be a transitional behavior on the pressure surface (Fig. 6(b)). It starts around the mid-chord in a 2D wave mode, followed by a 3D streak pattern of streamwise vortical structures. A detailed analysis of the transitional behavior is beyond the present scope. The emphasis here is on the scale-resolving capability and its mesh dependency. The numerical consistency has been clearly illustrated by the results of the two meshes with two time-step sizes, respectively.

The time-averaged surface pressure distributions computed with the two different mesh and time-step sizes, respectively, are compared in Fig. 7. The overall computed results compare well with the experimental data [38]. There are some detailed parts where certain mesh dependency is visible. Around the leading-edge, there is a suction surface spike, for which the different local resolutions of the two meshes are identifiable, similar to the results for this case by a different flow solver [28]. The effect of the computed leading-edge spike seems rather local. The detailed pressure distributions around the separation-transitional flow region also exhibit some dependency on local mesh resolution. A sharper rise of

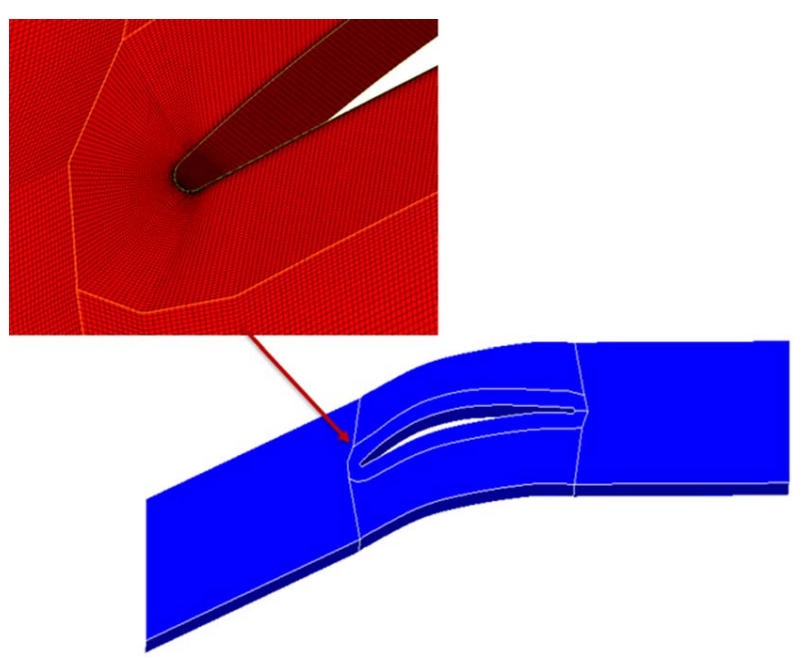

Fig. 5 Single-passage computational domain

Table 1 Numerical setup for sensitivity study

\begin{tabular}{|c|c|c|c|c|}
\hline \multirow{2}{*}{$\begin{array}{l}\text { Mesh density } \\
\text { Snanwise }\end{array}$} & \multicolumn{2}{|c|}{$\begin{array}{c}\text { Mesh } 1\left(3 \times 10^{6} / 10 \%\right. \\
\text { span })\end{array}$} & \multicolumn{2}{|c|}{$\begin{array}{c}\text { Mesh } 2\left(5 \times 10^{6} / 10 \%\right. \\
\text { span })\end{array}$} \\
\hline & \multirow{2}{*}{\multicolumn{2}{|c|}{$\begin{array}{l}32 \text { mesh cells } \\
800 \text { (both SS and PS) }\end{array}$}} & \multirow{2}{*}{\multicolumn{2}{|c|}{$\begin{array}{l}40 \text { mesh cells } \\
900 \text { (both SS and PS) }\end{array}$}} \\
\hline Chordwise & & & & \\
\hline Near-wall & $y_{\text {mean }}^{+}=0.96$ & $y_{\max }^{+}=3.5$ & $y_{\text {mean }}^{+}=0.91$ & $y_{\max }^{+}=3.5$ \\
\hline Time-step & $1 \mu \mathrm{s}$ & $0.5 \mu \mathrm{s}$ & $1 \mu \mathrm{s}$ & $0.5 \mu \mathrm{s}$ \\
\hline
\end{tabular}




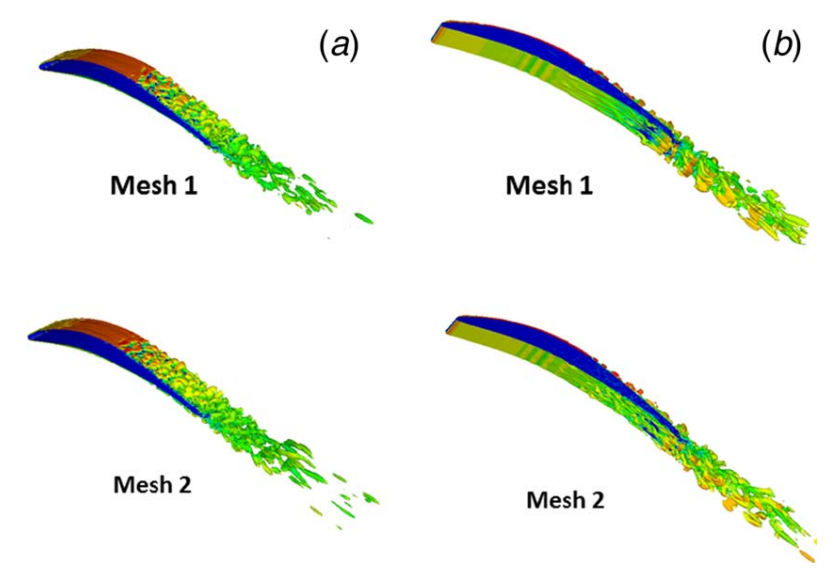

Fig. 6 Iso-surface of Q-criterion (Mesh 1 versus. Mesh 2) (isosurfaces are colored with axial velocity contours): (a) suction surface view and $(b)$ pressure surface view

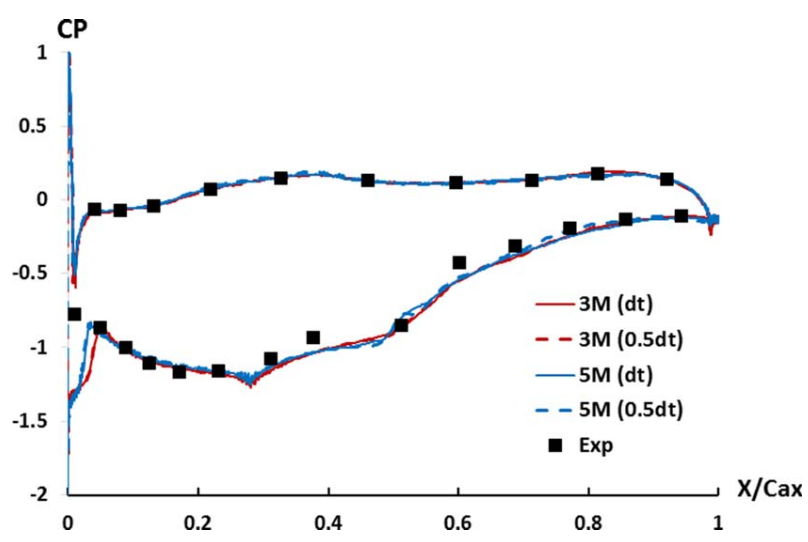

Fig. 7 Blade surface time-average pressures

pressure is observed for the finer mesh (Mesh 2) than that of the coarse one (Mesh 1), as expected. Overall, the mesh resolution of Mesh 1 (i.e., the $3 \times 10^{6}$ mesh cells for a $10 \%$ span sector) seems able to provide sufficient scale-resolving capability for the present work.

The results of this case serve to illustrate the LES capability of the flow solver adopted and also indicate a reasonable range of mesh density and time-step size for scale-resolving turbulent flow simulations of the present work

4.2 Multi-Passage Blade Row With Inlet Distortion. We now examine the capability of the present two-domain coupled solution for multiple blade passages. The global multi-passage domain is discretized with a coarse mesh. The embedded fine-mesh is used only for a single passage. For validation purposes, a reference solution is obtained for comparison where all passages of the multi-passage domain are embedded with the fine-mesh. This reference solution (denoted as "Direct Solution") will be compared to the coupled two-domain two-scale solution (denoted as "Two-scale Solution"). In the present work, the number of passages covered needs to be small enough, so that the direct fine-mesh solution can be obtained practically within a reasonable time (e.g., months rather than years). Here, a domain of six blade passages is taken.

Figure 8 shows the computational domain of the direct solution, where the near-wall regions of all six passages are refined. The blade geometry is the same as that used in the previous case. The base mesh however is rather coarse, with only five mesh points in the spanwise direction and 201 mesh points around the blade at

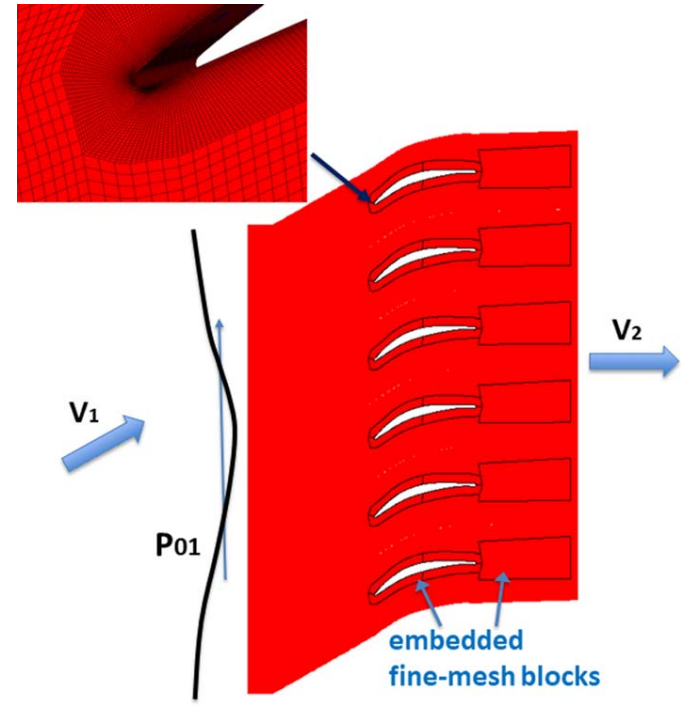

Fig. 8 Multi-passage domain (direct solution) (all passages are embedded with fine-mesh blocks)

each spanwise section. The near-wall fine-mesh blocks are generated by embedding $3 \times 3 \times 8$ mesh cells in each base-mesh cell, in the wall-tangential, the wall-normal, and the spanwise directions, respectively. The embedded fine-mesh density, indicated by the leading-edge close-up in Fig. 8, is taken with the local fine-mesh resolution comparable to that used in the previous test case, i.e., 33 mesh points in the spanwise direction for $10 \%$ span and 601 streamwise mesh points to cover both blade suction and pressures surfaces. The mesh density ratio between the embedded fine-mesh and the base coarse-mesh is 72 .

For the coupled two-scale solution, only is the first blade passage (the bottom passage in Fig. 8) embedded with the fine-mesh blocks. The other five passage domains all simply have the base coarse mesh.

The six-passage domain is subject to an inlet stagnation pressure distortion in a traveling wave form of

$$
P_{01}=\overline{P_{01}}\left[1+A_{p 01} \cos \left(\omega t-\varphi_{y}\right)\right], \quad\left(\varphi_{y}=2 \pi \frac{y}{6 Y_{p}}\right)
$$

where $y$ is the pitchwise distance and $Y_{p}$ is the blade pitch. The amplitude is taken to correspond to $7 \%$ inlet dynamic head. To simulate a stationary distortion in the absolute frame of reference, the distortion is relatively moving in a circumferential traveling waveform in the rotor frame of reference. The frequency of the traveling wave is set to correspond to a fixed non-moving distortion in the stator frame.

Moving to the results, we first take a look at the direct solution. Although the fine-mesh is only embedded in the near-wall and wake regions (Fig. 8), the scale-resolving capability seems to be well maintained. Figure 9 shows the instantaneous contours of the entropy function $\mathrm{e}^{(-\Delta \mathrm{S} / \mathrm{R})}$ at a temporal phase $\omega t=0$. The entropy rise $\Delta S$ is measured relative to the inlet mean state and $R$ the gas constant.

In Fig. 10, the contours of instantaneous unsteady vorticity component on the mid-span plane at $\omega t=0$ are compared among the three solutions. The direct solution (Fig. 10(a)) where all the six passages are embedded with the fine-mesh blocks provides a benchmarking reference. For the two-scale solution (Fig. 10(b)), only is the bottom blade-passage embedded with the fine-mesh blocks (as indicated in Fig. 2(b)). The URANS solution with the $\mathrm{S}-\mathrm{A}$ model for turbulence closure is obtained on the base coarse mesh (Fig. 10(c)). It is clear that the URANS solution hardly captures the unsteady vortex shedding around the trailing 


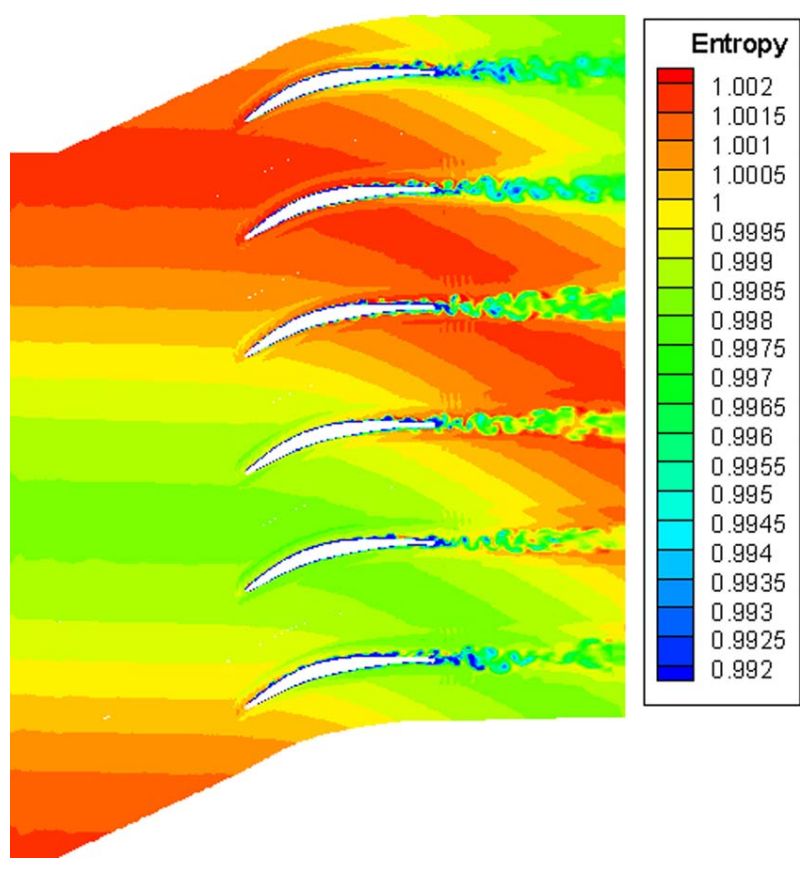

Fig. 9 Contours of unsteady entropy function, $e^{(-\Delta S / R)}$

edge, with the modeling consistency limited by the "doublecounting" effect of the modeled eddy-viscosity in the upstream boundary layer.

Comparing the two-scale solution with the direct solution, we can see that the embedded fine-mesh in the single-passage domain provides the desired scale-resolving capability locally (as shown in the passage at the bottom of Fig. 10(b)). On the other hand, the coarsemesh regions of the other five passages are clearly shown to be under-resolved, typified by the large-scale oscillatory wakes in these five passages. The unsteady flow pattern and scales of the five coarse-mesh regions are clearly different from those of the finemesh passage solution. For the present two-scale method, the key role of the source terms generated from the single-passage finemesh domain is to drive the unsteady solution of the coarse-mesh regions, with the end aim that the time-averaged coarse-mesh solution of these passages approaches that of the fine-mesh passage. In doing so, the final single-passage fine-mesh solution will also get being subject to a corrected environment at the coarsemesh resolved level.

Figure 11 compares the time-averaged axial velocities of the three solutions. The direct solution with all six passages embedded with the fine mesh (Fig. 11(a)) and the two-scale one with only single-passage embedded with the fine-mesh (Fig. 11(b)) agree well with each other, whilst the URANS solution (Fig. 11(c)) produces much thinner wakes due to lack of mixing.

The time-averaged axial velocities from a pitchwise traverse at $50 \%$ chord downstream of the blade trailing-edge are presented in Fig. 12. They are normalized by the average bulk velocity in the mainly inviscid flow region of the direct solution. The calculated velocity profiles illustrate that the present two-scale method with the local embedded fine-mesh for only one-passage can effectively and accurately provide the space-time averaged source terms. Once added with the source terms, the global coarse-mesh domain is enabled to produce a higher fidelity solution equivalent to having a fine-mesh locally.

4.3 Transonic Turbine Stage. Some basic validation of the present LES solver for a single-row turbine blade configuration has been recently carried out for the NASA C3X turbine cascade [39], as presented in Ref. [40]. Here, the focus is on assessing and demonstrating the multiple blade row capability of the present method. To this end, a high-pressure transonic turbine stage is examined. The geometry and flow conditions are similar to those used previously by the author [15].

A 2D view of a sector of $10 \%$ span is shown in Fig. 13. In order to carry out the direct fine-mesh solution, a configuration of three stator and five rotor passages is taken. The fine-mesh blocks are embedded in near-wall regions for both the NGV and the rotor blades except for the frontal part of the nozzle guise vane (NGV) pressure surface (as indicated in Fig. 13). Further fine-mesh blocks are added to the NGV region downstream of the trailing edge to better capture the vortex shedding. For each base coarsemesh cell, $4 \times 4 \times 10$ fine-mesh cells are embedded in the walltangential, wall-normal, and spanwise directions, respectively. Thus, the mesh density ratio between the fine and coarse meshes is 160 .
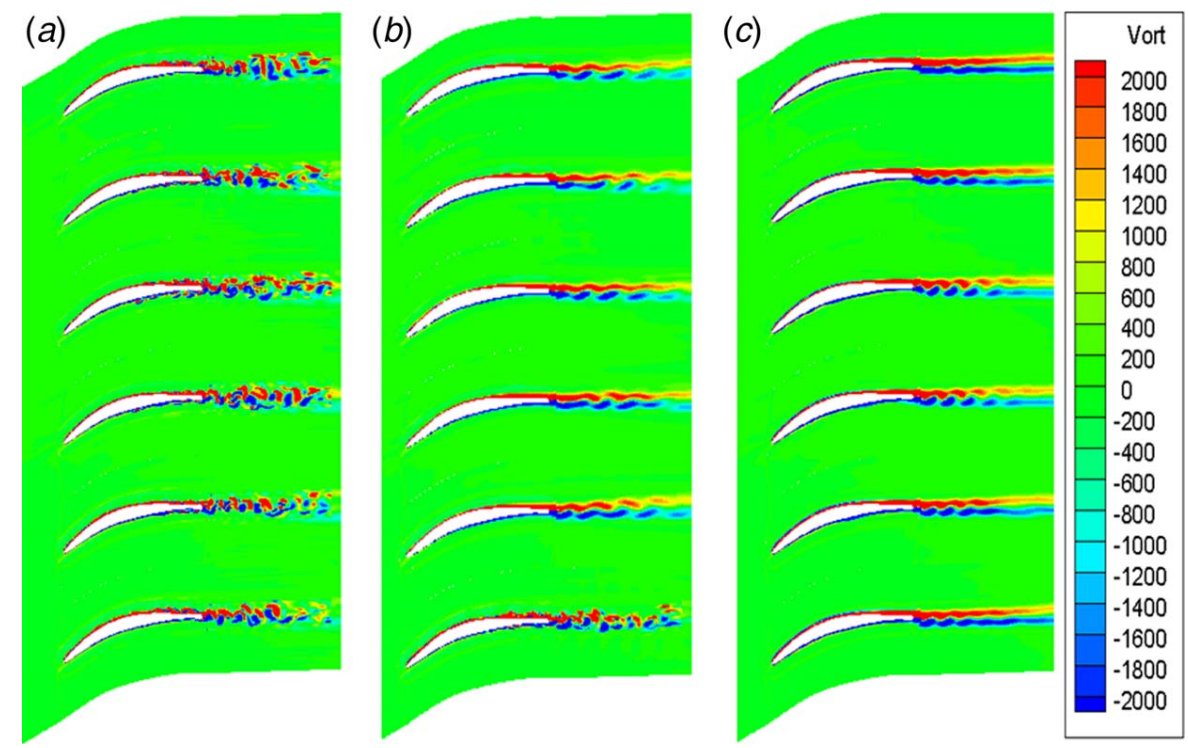

Fig. 10 Instantaneous vorticity (spanwise component): (a) direct solution, (b) two-scale solution, and (c) URANS 

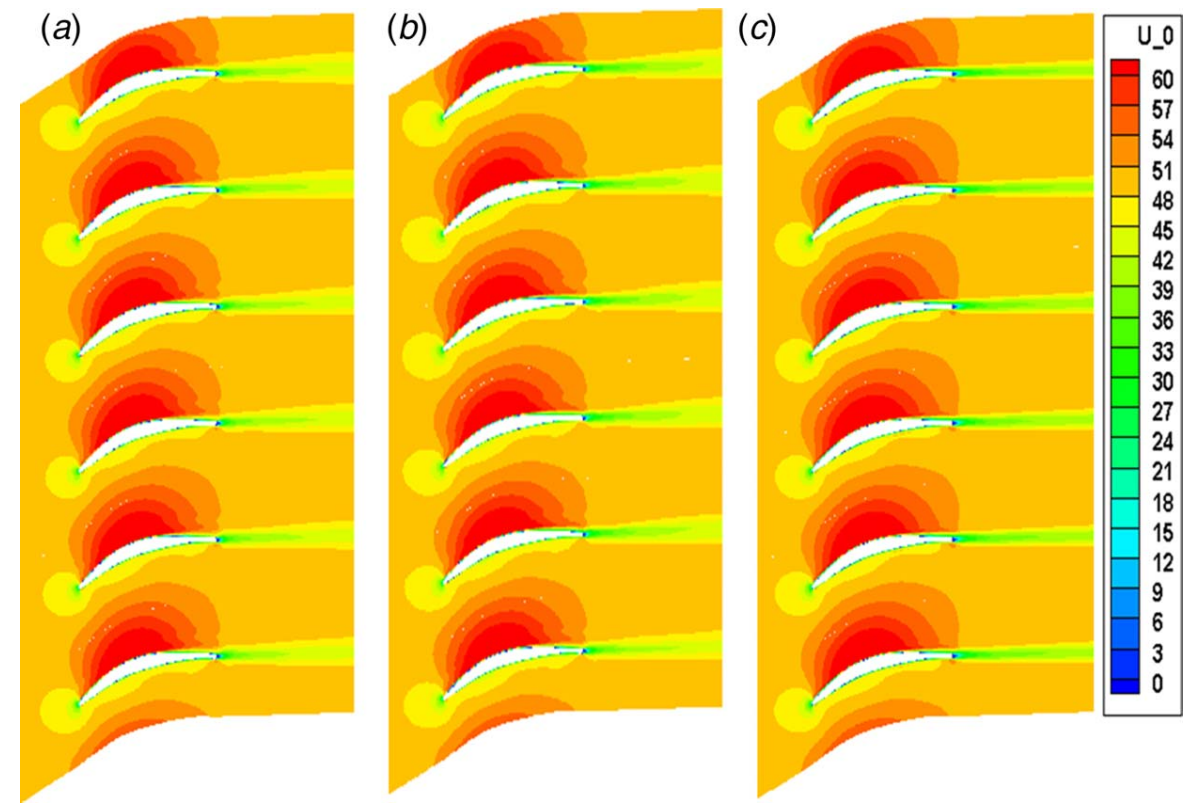

Fig. 11 Time-averaged axial velocity: (a) direct solution, (b) two-scale solution, and (c) URANS

For the two-scale solution, only is a single passage for each blade row embedded with the fine mesh (Fig. 13). For the direct solution, all blade passages for each row are embedded in the same way. The unsteady solutions are carried out in the dual timing mode with a physical time-step of $0.2 \mu \mathrm{s}$, and 1000 time-steps are needed for one NGV passing period "seen" by a rotor blade.

Figure 14 compares the instantaneous entropy contours among the direct solution, the two-scale solution, and the URANS (the S-A model). The coarse-mesh solution with the propagated source terms does not resolve the unsteady flow field as well as in those embedded fine-mesh regions, which is easily identifiable by comparing the NGV wakes between the middle passage (coarsemesh) and the bottom one (embedded fine-mesh), as shown in Fig. 14(b). This low unsteady resolution is expected, as the source terms are only meant to drive the coarse-mesh region to match the target space-time averaged flow, as intended.

The time-average entropy function contours are compared in Fig. 15. The results consistently demonstrate the intended modeling effect. This case is in a highly loaded off-deign setting due to the changed blade count adopted. The URANS solution produces very thick and persistent wakes without strong turbulence mixing indicated (Figs. 14(c) and 15(c)). On the other hand, the turbulence enhanced mixing of the NGV wakes is well captured by the two-scale solution (Figs. 14(b) and $15(b)$ ) in agreement with the direct solution (Figs. 14(a) and 15(a)).

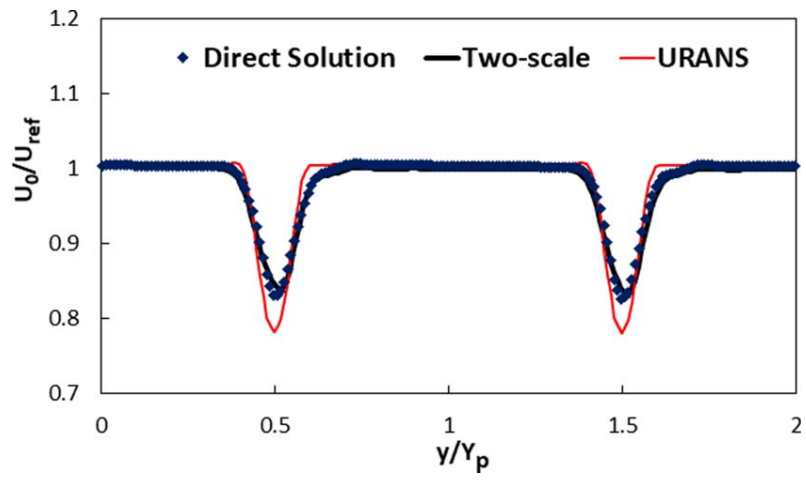

Fig. 12 Pitchwise traverse of time-average axial velocity
For the rotor row, it is of interest to note that in the URANS solution, a markedly high loss (corresponding to the low value of the entropy function) region shows up close to the blade suction surface, whilst the opposite exists near the pressure surface side within a rotor passage (Fig. 15(c)). This is likely to be attributed to the "negative jetting" effect (or "wake dispersion" [41]) of the NGV wakes seen in the rotor passage. The velocity deficit within a moving NGV wake effectively convects the lossy fluid relatively from the pressure surface to the suction surface (Fig. 14(c)), with a net effect of low time-mean loss on the pressure side and high loss on the suction side in rotor passages (Fig. 15(c)). In contrast to the URANS solution, both the LES solutions show much weaker net

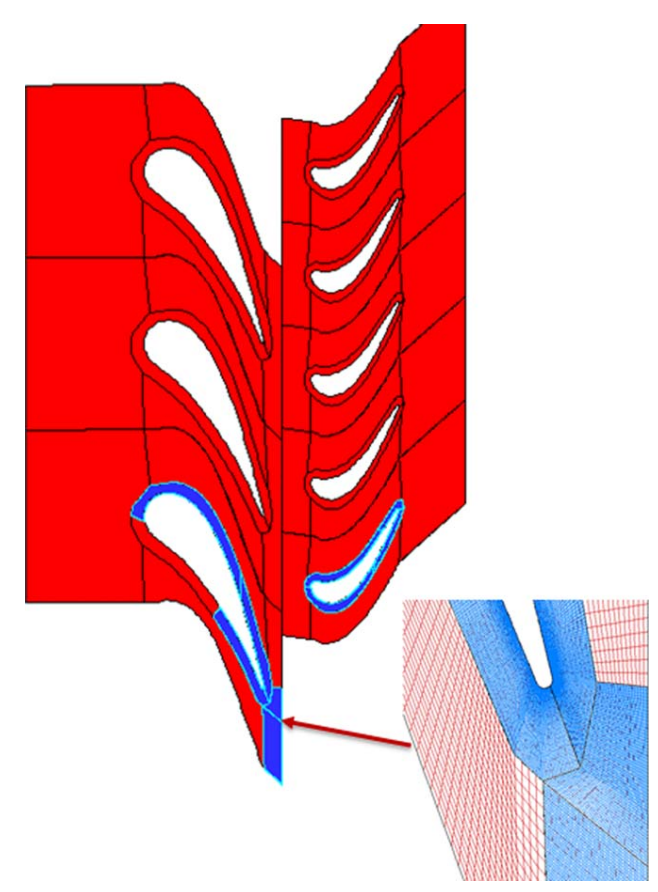

Fig. 13 Computational domain for turbine stage (dark-colored regions are embedded with fine mesh) 

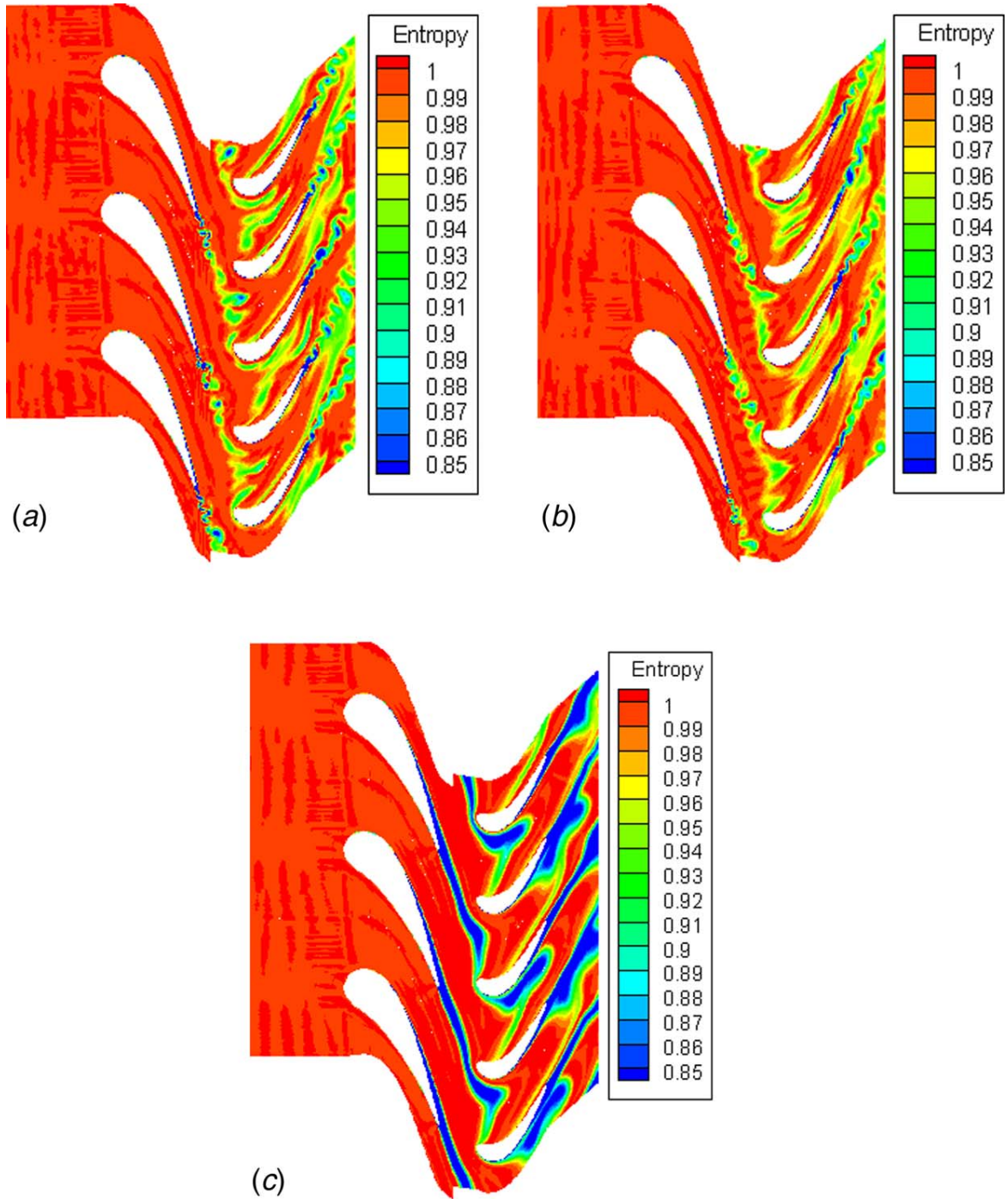

Fig. 14 Unsteady entropy function, $e^{(-\Delta S / R)}$ : (a) direct solution, (b) two-scale solution, and (c) URANS

cross-passage entropy migration within the rotor passages. This should be reasonably expected given the strong mixing of the NGV wakes before reaching the rotor row as seen in the LES solutions (Figs. 14(a) and 14(b)). Consequently, much weakened NGV wakes due to strong turbulence mixing in the LES results should lead to a much weaker "negative jetting" behavior in the rotor passages.

More detailed comparisons are given in terms of the pitchwise traverses of the time-average entropy function at the NGV side of the stator-rotor interface (Fig. 16(a)) and at the rotor domain exit (Fig. 16(b)). At the NGV exit, the effect of the under-mixing in the coarse-mesh region of the two-scale solution, though much weaker than that of the URANS, can still be seen (Fig. 16(a)). For the rotor exit traverse, we can see a clear shift of the high loss core from the wake position relatively to the suction side in the URANS results (Fig. 16(b)), which is consistent with the strong "negative jetting" behavior in the URANS solution as discussed earlier.

Finally, a stage configuration with 10 stator and 19 rotor passages is taken to illustrate the potential effectiveness of the method for more realistic larger blade counts. The instantaneous and timeaveraged entropy contours of the overall domain configuration are shown in Fig. 17. The corresponding closeups of those blade passages with embedded fine meshes are shown in Fig. 18. The fine-mesh blocks are embedded in the base coarse mesh in the near- wall and wake regions of a single-passage for each row $(4 \times 4 \times 8$ fine-mesh cells embedded in each coarse-mesh cell). The comparison in unsteady entropy contours between the two fine-mesh passages and other neighboring coarse-mesh passages clearly illustrates the differences in mesh resolution between the base coarse-mesh and embedded fine mesh (Fig. 18(a)). On the other hand, the time-averaged flow of those coarse-mesh passages agrees well that of the single passage embedded with the fine-mesh (the bottom passage in Fig. 18(b)), underlining again the intended outcome of the present approach.

All the test cases consistently demonstrate that the present averaging-based two-scale method is able to provide a significant improvement in terms of the flow modeling fidelity. It must be noted that the main objective of the present work is to examine the validity of the method so that the numbers of the blade passages taken need to be small in order to get a corresponding direct solution for comparison purposes within a reasonable length of time. For a realistic blade count, there will have to be significantly larger numbers of passages. The cost of a direct solution will have to scale with the number of blade passages accordingly, but the cost of the present two-scale solution should be largely the same. Therefore, the method has the potential for a scale-resolving solution with equivalently high fidelity but at a fractional cost of a conventional direct multi-passage fine-mesh solution. 

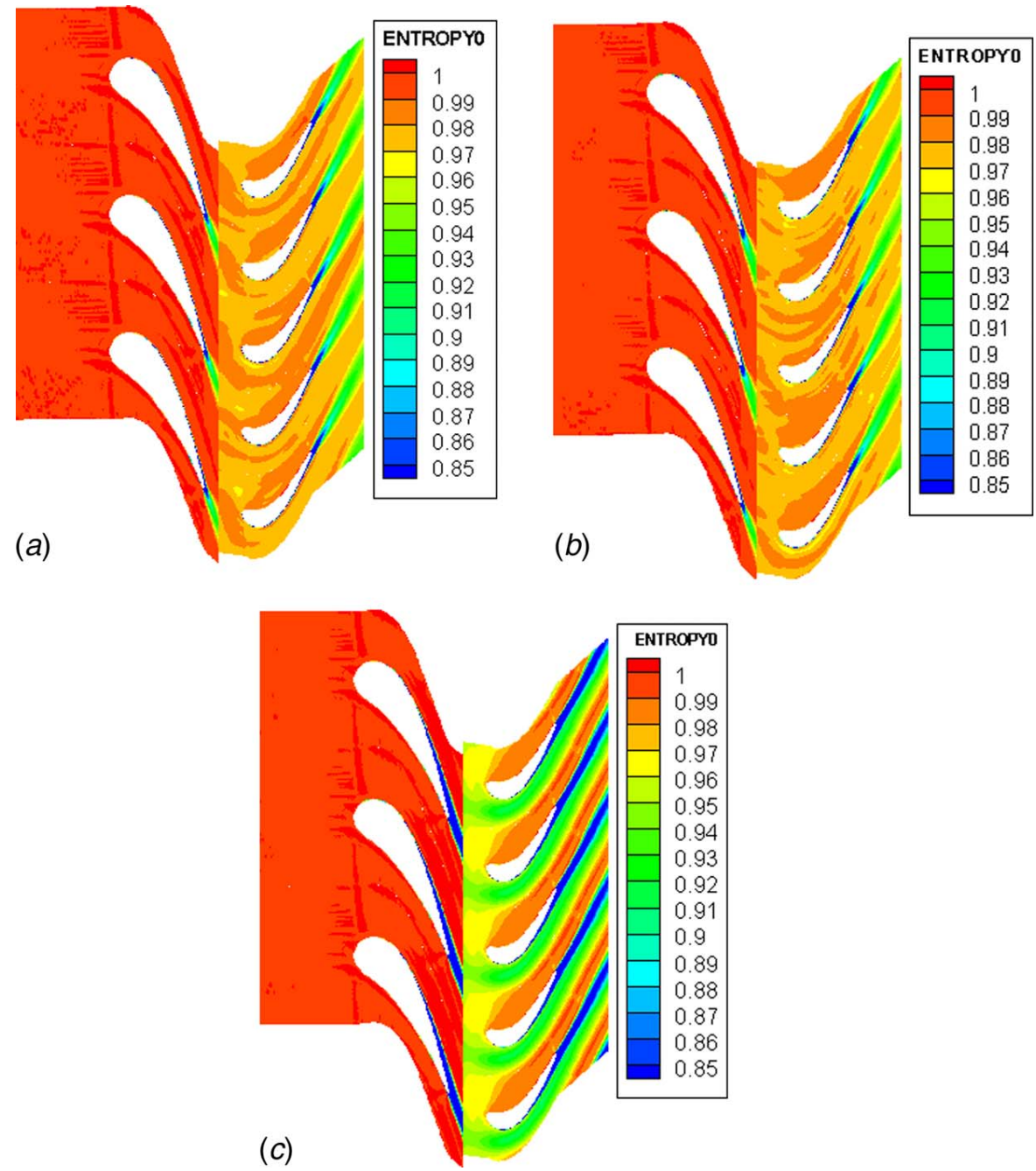

Fig. 15 Time-average entropy function, $e^{(-\Delta S O / R)}$ : (a) direct solution, (b) two-scale solution, and (c) URANS
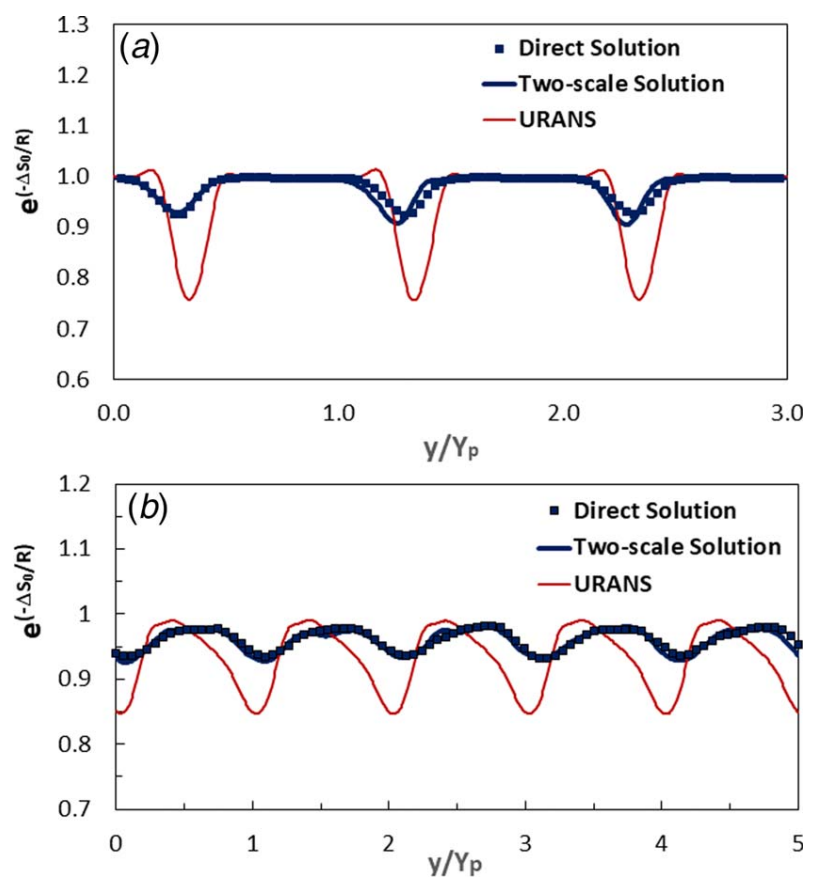

Fig. 16 Pitchwise traverses of time-average loss: (a) NGV exit and $(b)$ rotor exit

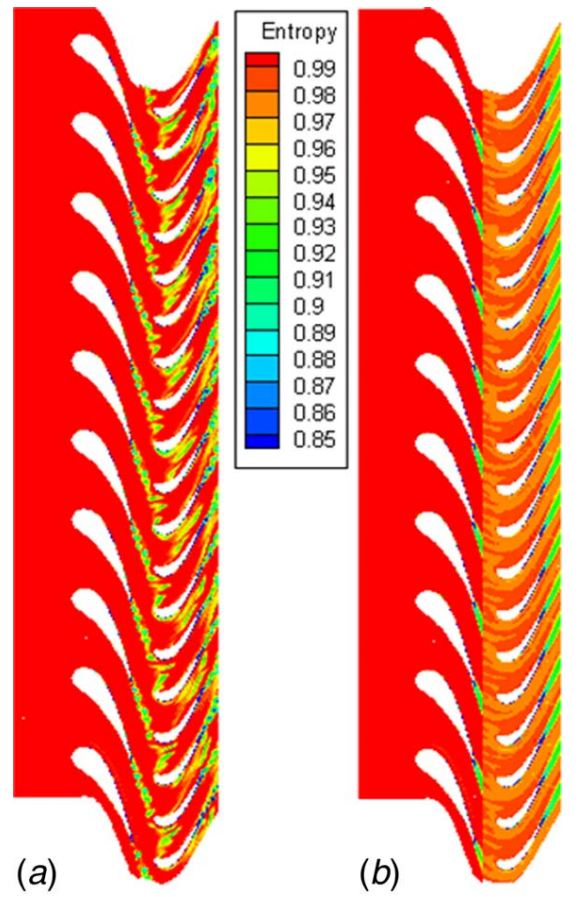

Fig. 17 Contours of entropy function, $e^{(-\Delta S / R)}$ : (a) unsteady and (b) time-averaged 


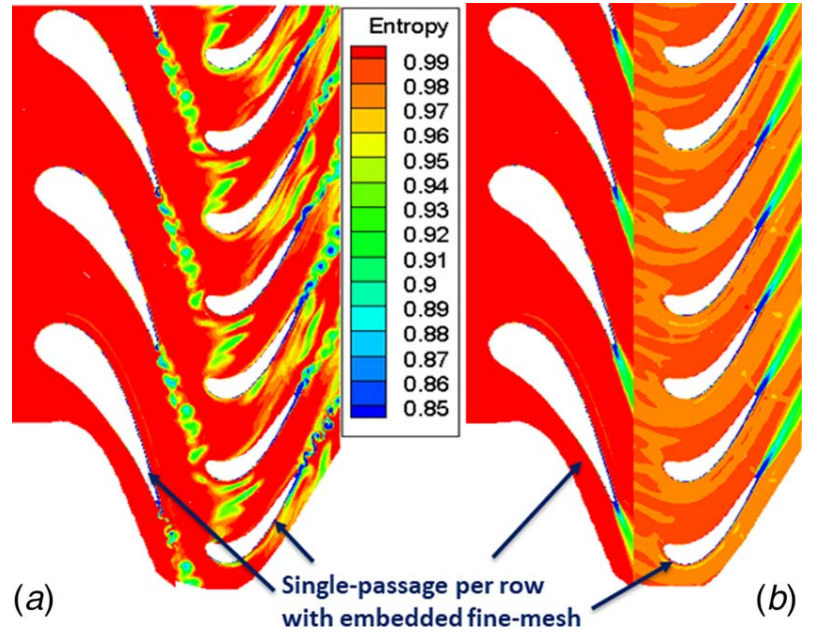

Fig. 18 Contours of entropy function, $e^{(-\Delta S / R)}$ (around embedded passages): (a) unsteady and (b) time-averaged

\section{Concluding Remarks}

The basic intent is to establish and capture the interaction between two very contrasting scenarios: a local (single-passage) fine-mesh domain with high resolution but uncertain surrounding/ boundary conditions, and a global (multi-passage) coarse-mesh domain with well-defined boundary conditions but inadequate resolution. There are two key aspects of the framework serving as the linkage between the two domains. First, a locally spatially averaged fine-mesh solution, when projected to corresponding coarse-mesh cells, can be effectively used as the target for the global coarse-mesh counterpart. Second, the extra product terms due to averaging over a nonlinear process, in space as well as in time, can be used as a source to drive the global coarse-mesh domain solution to its target counterpart. The considerations lead to a space-time averaged and source-terms-based two-scale methodology, as instigated, implemented, and demonstrated in the present work.

From the perspectives of both the basic framework concept and the practical implementation, the working of the present method benefits from casting the space-time averaging in either a direct mode or an inverse mode, in analogy to the Reynolds-averaging in time. Lumping all the extra nonlinear product terms together leads to a compact form of the source terms (one scalar for one equation). The source terms can then be obtained simply by a noniterative evaluation of total net-flux residuals using the target flow variables. Given the passage-passage similarity in the timeaveraged flow, the source terms obtained can be directly mapped to a multi-passage coarse-mesh domain, effectively capturing the interaction between the two domains. A converged solution of this coupled two-scale system should meet two seemingly conflicting requirements: an otherwise poorly conditioned local fine-mesh domain is now subject to a right environment/flow condition, and an otherwise poorly resolved global coarse-mesh domain is now effectively subject to a higher resolution.

Computational test cases with up to two orders of magnitude local fine/coarse-mesh density ratios are examined. The results consistently demonstrate the validity of the present framework, methodology, and implementation. Further validations and applications are expected.

\section{Acknowledgment}

The author is grateful to Professor John Denton for the profound influence of his "integral" approaches to complex problems. Denton's simple form of lumped viscous terms, regardless of modeling fidelities adopted, still seems to be as insightfully relevant today as it was more than 30 years ago.

\section{Conflict of Interest}

There are no conflicts of interest.

\section{Data Availability Statement}

The data sets generated and supporting the findings of this article are obtainable from the corresponding author upon reasonable request. The authors attest that all data for this study are included in the paper.

\section{Nomenclature}

$S=$ source term vector with five elements

$U=$ flow variable vector with five elements

$\bar{U}=$ time-averaged flow variable

$\tilde{U}=$ local spatially averaged variable (volume-weighted average of fine-mesh variables over a coarse-mesh cell)

$\overline{\tilde{U}}=$ space-time averaged flow variable

$S_{t}=$ source term for time-averaging (Reynolds-averaging)

$S_{s t}=$ source term for space-time averaging

$U_{C}=$ flow variable of a coarse-mesh domain

$U_{f}=$ flow variable of a fine-mesh domain

$Y_{p}=$ blade pitch length

\section{References}

[1] Denton, J. D., 1975, “A Time Marching Method for Two and Three Dimensional Blade to Blade Flows," ARC R\&M 3775.

[2] Denton, J. D., 1986, "The Use of a Distributed Body Force to Simulate Viscous Flow in 3D Flow Calculations," ASME Paper 86-GT-144.

[3] Hah, C., 1987, "Calculation of Three-Dimensional Viscous Flows in Turbomachinery With an Implicit Relaxation Method,” J. Propul. Power, 3(5), pp. 415-422.

[4] Dawes, W. N., 1988, "Development of a 3D Navier-Stokes Solver for Application to All Types of Turbomachinery," ASME Paper 88-GT-70.

[5] Spalart, P. R., and Allmaras, S. R., 1992, "A One-Equation Turbulence Model for Aerodynamic Flows," AIAA Paper, 92-0439.

[6] Menter, F. R., 2009, "Review of the Shear-Stress Transport Turbulence Model Experience From an Industrial Perspective," Int. J. Comput. Fluid Dyn., 23(34).

[7] Denton, J. D., and Singh, U. K., 1979, "Time-marching Methods for Turbomachinery Flow Calculations," VKI -LEC-SER-1979-7, Von Karman Institute for Fluid Dynamics, Belgium.

[8] Denton, J. D., 1992, "The Calculation of Three-Dimensional Viscous Flow Through Multistage Turbomachine," ASME J. Turbomach., 114(1), pp. 18-26.

[9] Adamczyk, J. J., 1985, "Model Equations for Simulating Flows in Multistage Turbomachinery," ASME Paper 85-GT-226.

[10] Adamczyk, J. J., 2000, "Aerodynamic Analysis of Multistage Turbomachinery Flows in Support of Aerodynamic Design,” ASME J. Turbomach., 122(3).

[11] He, L., and Denton, J. D., 1994, "Three Dimensional Time-Marching Inviscid and Viscous Solution for Unsteady Flows Around Vibrating Blades," ASME J. Turbomach., 116(3), pp. 469-476.

[12] Vahdati, M., and Imregun, M., 1996, "A Non-linear Aeroelasticity Analysis of a Fan Blade Using Unstructured Dynamic Meshes," Proc. Inst. Mech. Eng. Part C: J. Mech. Eng. Sci., 210(6), pp. 549-564.

[13] Clark, W. S., and Hall, K. C., 2000, "A Time-Linearised Navier-Stokes Analysis of Stall Flutter,” J. Turbomach., 122(3), pp. 467-476.

[14] Dorney, D., Davis, R., Edwards, D., and Madhavan, N., 1992, "Unsteady Analysis of Hot-Streak Migration in a Turbine Stage," J. Propul. Power, 8(2), pp. 520-529.

[15] He, L., Menshikova, V., and Haller, B. R., 2007, "Effect of Hot-Streak Counts on Turbine Blade Heat Load and Forcing," AIAA J. Propul. Power, 23(6).

[16] Ong, J., Miller, R. J., and Denton, J. D., 2010, "The Prediction of Hot Streak Migration in a High-Pressure Turbine," Proc. Inst. Mech. Eng. Part A: J. Power Energy, 224.

[17] Pullan, G., Young, A. M., Day, I. J., Gretizer, E. M., and Spakovszky, Z. S., 2015, "Origins and Structure of Spike-Type Rotating Stall," J. Turbomach., 137(5).

[18] Zhang, L. Y., He, L., and Stueer, H., 2013, "A Numerical Investigation of Rotating Instability in Steam Turbine Last Stage," ASME J. Turbomach., 135(1), p. 011009.

[19] Ning, W., and He, L., 2001, "Some Modelling Issues on Trailing Edge Vortex Shedding," AIAA J., 39(5), pp. 787-793.

[20] Tucker, P. G., Eastwood, S., Klostermeier, C., Jefferson-Loveday, R., Tyacke, J., and Liu, Y., 2011, "Hybrid LES Approach for Practical Turbomachinery FlowsPart I: Hierarchy and Example Simulation," J. Turbomach., 134(2).

[21] Memory, C. L., Chen, J. P., and Bons, J. P., 2016, "Implicit Large Eddy Simulation of a Stalled Low Pressure Turbine Airfoil," ASME J. Turbomach., 138(7), p. 071008 .

[22] Gourdain, N., Sicot, F., Duchaine, F., and Gicquel, L., 2014, "Large Eddy Simulation of Flows in Industrial Compressors: A Path From 2015 to 2035," Philos. Trans. R. Soc. A., 372(2022), p. 20130323. 
[23] Michelassi, V., Chen, L.-W., Pichler, R., and Sandberg, R. D., 2015 , "Compressible Direct Numerical Simulation of Low-Pressure Turbines-Part II: Effect of Inflow Disturbances," ASME J. Turbomach., 137(7), p. 071005.

[24] Wheeler, A. P. S., Sandberg, R. D., Sandham, N. D., Pichler, R., Michelassi, V., and Laskowski, G., 2016, "Direct Numerical Simulations of High Pressure Turbine Vane,” ASME J. Turbomach., 138(7), p. 071003.

[25] Spalart, P. R., 2009, "Detached-Eddy Simulation," Ann. Rev. Fluid Mech., 41(1), pp. 181-202.

[26] Ling, J., and Templeton, J., 2015, "Evaluation of Machine Learning Algorithms for Prediction of Regions of High Reynolds Averaged Navier-Stokes Uncertainty," Phys. Fluids, 27(8), p. 085103

[27] Wetheritt, J., and Sandberg, R. D., 2017, "The Development of Algebraic Stress Models Using a Novel Evolutionary Algorithm," Int J. Heat Fluid Flow, 68

[28] He, L., and Yi, J., 2015, "Two-scale Methodology for URANS / Large Eddy Simulation Solutions of Unsteady Turbomachinery Flows," J. Turbomach., 139(10).

[29] Cumpsty, N. A., and Horlock, J. H., 2006, “Averaging Nonuniform Flow for a Purpose," J. Turbomach., 128(1), pp. 120-129.

[30] He, L., 1993, "New Two-Grid Acceleration Method for Unsteady Navier-Stokes Calculations," AIAA J. Propul. Power, 9(2), pp. 272-280.

[31] He, L., 2008, "Harmonic Solution of Unsteady Flow Around Blade With Separation," AIAA J., 46(6).

[32] He, L., 2018, "Multiscale Block Spectral Solution for Unsteady Flows," Int. J. Numer. Methods Fluids, 86(10), pp. 655-678.
[33] Liou, M.-S., 2006, “A Sequel to AUSM, Part II: AUSM+-up for All Speeds, An Accurate and Robust Flux Splitting Scheme for Shock and Contact Discontinuities," J. Comput. Phys., 214(1), pp. 137-170.

[34] Cada, M., and Torrilhon, M., 2009, "Compact Third-Order Limiter Functions for Finite Volume Methods,” J. Comput. Phys., 228(11), pp. 4118-4145.

[35] Jameson, A., 1991, "Time-Dependent Calculations Using Multi-Grid, With Applications to Unsteady Flows Past Airfoil and Wings," AIAA Paper 91-1596.

[36] He, L., 2006, "Fourier Modeling of Steady and Unsteady Nonaxisymmetrical Flows," J. Propul. Power, 22(1), pp. 197-201.

[37] Sanger, N. L., and Shreeve, R. P., 1986, "Comparison of Calculated and Experimental Cascade Performance for Controlled-Diffusion Compressor Stator Blading," ASME J. Turbomach., 108(1), pp. 42-50.

[38] Yang, H., and He, L., 2004, "Experimental Study on Linear Compressor Cascade With Three-Dimensional Blade Oscillation," AIAA J. Propul. Power, 20(1), pp. $180-188$.

[39] Hylton, L. D., Mihelc, M. S., Turner, E. R., Nealy, D. A., and York, R. E., 1983, "Analytical and Experimental Evaluation of the Heat Transfer Distribution Over the Surface of Turbine Vanes," NASA Technical Report No. CR 168015.

[40] He, L., 2019, "Closely Coupled Fluid-Solid Interface Method With Moving-Average for LES Based Conjugate Heat Transfer Solution," Int. J. Heat Fluid Flow, 79, p. 10840.

[41] Smith, L. H., 1966, "Wake Dispersion in Turbomachines," J. Basic Eng. ASME Trans., 88. Ser. D, No. 3. 